TRANSACTIONS OF THE

AMERICAN MATHEMATICAL SOCIETY

Volume 360, Number 5, May 2008, Pages 2285-2301

S 0002-9947(07)04245-6

Article electronically published on December 11, 2007

\title{
GIAMBELLI FORMULAE FOR THE EQUIVARIANT QUANTUM COHOMOLOGY OF THE GRASSMANNIAN
}

\author{
LEONARDO CONSTANTIN MIHALCEA
}

\begin{abstract}
We find presentations by generators and relations for the equivariant quantum cohomology of the Grassmannian. For these presentations, we also find determinantal formulae for the equivariant quantum Schubert classes. To prove this, we use the theory of factorial Schur functions and a characterization of the equivariant quantum cohomology ring.
\end{abstract}

\section{INTRODUCTION}

Let $X$ denote the Grassmannian $G r(p, m)$ of subspaces of dimension $p$ in $\mathbb{C}^{m}$. One of the fundamental problems in the study of the equivariant quantum cohomology algebra of $X$ is to compute its structure constants, which are the 3-point, genus 0, equivariant Gromov-Witten invariants. The goal of this paper is to give a method for such a computation. Concretely, we realize the equivariant quantum cohomology as a ring given by generators and relations, and we find polynomial representatives (i.e. Giambelli formulae) for the equivariant quantum Schubert classes (which form a module-basis for this ring). ${ }^{1}$ These polynomials will be given by certain determinants which appear in the Jacobi-Trudi formulae for the factorial Schur functions (see $\S 2$ below for details).

Since the equivariant quantum cohomology ring specializes to both quantum and equivariant cohomology rings, we also obtain, as corollaries, determinantal formulae for the Schubert classes in the quantum and equivariant cohomology. In fact, in the quantum case, we recover Bertram's quantum Giambelli formula [3]. In the case of equivariant cohomology, we show that the factorial Schur functions represent the equivariant Schubert classes. The latter result, although not explicitly stated in the literature, seems to have been known before (cf. Remark 2 in $\S 5$ ).

We next recall some of the basic facts about the equivariant quantum cohomology and fix the notation. The torus $T \simeq\left(\mathbb{C}^{*}\right)^{m}$ acts on the Grassmannian $X$ by the action induced from the $G L(m)$-action. The $T$-equivariant cohomology of a point, denoted $\Lambda$, is the polynomial ring $\mathbb{Z}\left[T_{1}, \ldots, T_{m}\right]$ in the equivariant parameters $T_{i}$, graded by $\operatorname{deg} T_{i}=1$ (see $\S 5.1$ for a geometric interpretation of $T_{i}$ ). Let $q$ be an indeterminate of degree $m$. The $T$-equivariant quantum cohomology of $X$, denoted $Q H_{T}^{*}(X)$, is a graded, commutative, $\Lambda[q]$-algebra with a $\Lambda[q]$-basis $\left\{\sigma_{\lambda}\right\}$ indexed by partitions $\lambda=\left(\lambda_{1}, \ldots, \lambda_{p}\right)$ included in the $p \times(m-p)$ rectangle

Received by the editors June 17, 2005 and, in revised form, November 9, 2005.

2000 Mathematics Subject Classification. Primary 14N35; Secondary 05E05, 14F43.

${ }^{1}$ It is a standard fact that given a ring $R / I$, where $R$ is a polynomial ring and $I$ a homogeneous ideal, together with some elements in $R$ which determine a module-basis for $R / I$, the structure constants for this basis can be computed using e.g. Gröebner basis methods.

(C) 2007 American Mathematical Society Reverts to public domain 28 years from publication 
(i.e. $\lambda_{1}, \ldots, \lambda_{p}$ are integers such that $m-p \geqslant \lambda_{1} \geqslant \ldots \geqslant \lambda_{p} \geqslant 0$ ). This basis is determined by the Schubert varieties in $X$, defined with respect to the standard flag, and the classes $\sigma_{\lambda}$ will be called (equivariant quantum) Schubert classes. More details are given in $\S 3$ and especially in [23]. The equivariant quantum multiplication is denoted by $\circ$ and it is determined by the 3-pointed, genus 0 , equivariant Gromov-Witten invariants $c_{\lambda, \mu}^{\nu, d}$. In this paper we refer to these coefficients as the equivariant quantum Littlewood-Richardson coefficients, abbreviated EQLR. They have been introduced by Givental-Kim in [13] (see also [16, 12, 17]), together with the equivariant quantum cohomology. Then, by definition,

$$
\sigma_{\lambda} \circ \sigma_{\mu}=\sum_{d \geqslant 0} \sum_{\nu} q^{d} c_{\lambda, \mu}^{\nu, d} \sigma_{\nu}
$$

The EQLR coefficients $c_{\lambda, \mu}^{\nu, d}$ are homogeneous polynomials in $\Lambda$ of degree $|\lambda|+$ $|\mu|-|\nu|-m d$, where $|\alpha|$ denotes the sum of all parts of the partition $\alpha$. They are equal to the structure constants of equivariant cohomology if $d=0$ and to those of quantum cohomology (i.e. to the ordinary Gromov-Witten invariants) if $|\lambda|+|\mu|-|\nu|-m d=0$. Equivalently, the quotient of the equivariant quantum cohomology ring by the ideal generated by the equivariant parameters $T_{i}$ yields the quantum cohomology ring of $X$ (a $\mathbb{Z}[q]$-algebra), while the quotient by the ideal generated by $q$ yields the $T$-equivariant cohomology of $X$ (a $\Lambda$-algebra). More about the EQLR coefficients, including a certain positivity, which generalizes the positivity enjoyed by the equivariant coefficients, can be found in $[23,24]$.

1.1. Statement of the results. As we have noted before, the equivariant quantum Giambelli formula which we obtain is a "factorial" generalization of Bertram's quantum Giambelli formula [3], and, as in that case, it doesn't involve the quantum parameter $q$. It is closely related to a certain generalization of ordinary Schur functions, called factorial Schur functions. These are polynomials $s_{\lambda}(x ; t)$ in two sets of variables: $x=\left(x_{1}, \ldots, x_{p}\right)$ and $t=\left(t_{i}\right)_{i \in \mathbb{Z}}$. They play a fundamental role in the study of central elements in the universal enveloping algebra of $\mathfrak{g l}(n)([27,28,25])$. One of their definitions is via a "factorial Jacobi-Trudi" determinant, and this determinant will represent the equivariant quantum Schubert classes. The basic properties of the factorial Schur functions are given in $\S 2$.

Denote by $h_{i}(x ; t)$ (respectively by $e_{j}(x ; t)$ ) the complete homogeneous (respectively, elementary) factorial Schur functions. They are equal to $s_{(i)}(x ; t)$ (respectively $s_{(1)^{i}}(x ; t)$ ), where $(i)$ (respectively $\left.(1)^{i}\right)$ denotes the partition $(i, 0, \ldots, 0)$ (respectively $(1, \ldots, 1,0, \ldots, 0)$, with $i$ 's $)$. Let $t=\left(t_{i}\right)_{i \in \mathbb{Z}}$ be the sequence defined by

$$
t_{i}= \begin{cases}T_{m-i+1} & \text { if } 1 \leqslant i \leqslant m \\ 0 & \text { otherwise }\end{cases}
$$

where $T_{i}$ is an equivariant parameter. The next definitions are inspired from those used in the theory of factorial Schur functions. For an integer $s$ we define the shifted sequence $\tau^{s} t$ to be the sequence whose $i-$ th term $\left(\tau^{s} t\right)_{i}$ is equal to $t_{s+i}$. Let $h_{1}, \ldots, h_{m-p}$ and $e_{1}, \ldots, e_{p}$ denote two sets of indeterminates (these correspond to the complete homogeneous, respectively, elementary, factorial Schur functions). Often one considers shifts $s_{\lambda}\left(x \mid \tau^{s} t\right)$ of $s_{\lambda}(x \mid t)$ by shifting the sequence $\left(t_{i}\right)$. Corresponding to these shifts we define the shifted indeterminates $\tau^{-s} h_{i}$, respectively $\tau^{s} e_{j}$, where $s$ is a nonnegative integer, as elements of $\Lambda\left[h_{1}, \ldots, h_{m-p}\right]$, respectively $\Lambda\left[e_{1}, \ldots, e_{p}\right]$. 
The definition of $\tau^{-s} h_{j}$ is given inductively as a function of $\tau^{-s+1} h_{j}$ and of $\tau^{-s+1} h_{j-1}$, and it is modelled on an equation which relates $h_{j}\left(x \mid \tau^{-s} t\right)$ to $h_{j}\left(x \mid \tau^{-s+1} t\right.$ ) (see eq. (2.6) below, with $a:=\tau^{-s+1} t$ ). Concretely, $\tau^{0} h_{j}=h_{j}$, $\tau^{-1} h_{j}=h_{j}+\left(t_{j-1+p}-t_{0}\right) h_{j-1}$ and, in general,

$$
\tau^{-s} h_{j}=\tau^{-s+1} h_{j}+\left(t_{j+p-s}-t_{1-s}\right) \tau^{-s+1} h_{j-1} .
$$

Similarly, the definition of $\tau^{s} e_{i}$ is modelled on an equation which relates $e_{j+1}\left(x \mid \tau^{s} t\right)$ to $e_{j+1}\left(x \mid \tau^{s-1} t\right.$ ) (see eq. (2.7) below, with $a:=\tau^{s-1} t$ ), and it is given by

$$
\tau^{s} e_{i}=\tau^{s-1} e_{i}+\left(t_{s}-t_{p-i+s+1}\right) \tau^{s-1} e_{i-1}
$$

with $\tau^{0} e_{i}=e_{i}$. By convention, $h_{0}=e_{0}=1, h_{j}=0$ if $j<0$ or $j>m-p$, and $e_{i}=0$ if $i<0$ or $i>p$.

For $\lambda$ a partition in the $p \times(m-p)$ rectangle define $s_{\lambda} \in \Lambda\left[h_{1}, \ldots, h_{m-p}\right]$, respectively $\widetilde{s}_{\lambda} \in \Lambda\left[e_{1}, \ldots, e_{p}\right]$, analogously to the definition of the factorial Schur function $s_{\lambda}(x \mid t)$, via the factorial Jacobi-Trudi determinants (cf. (2.4) below):

$$
\begin{gathered}
s_{\lambda}=\operatorname{det}\left(\tau^{1-j} h_{\lambda_{i}+j-i}\right)_{1 \leqslant i, j \leqslant p}, \\
\widetilde{s}_{\lambda}=\operatorname{det}\left(\tau^{j-1} e_{\lambda_{i}^{\prime}+j-i}\right)_{1 \leqslant i, j \leqslant m-p} .
\end{gathered}
$$

Here $\lambda^{\prime}=\left(\lambda_{1}^{\prime}, \ldots, \lambda_{m-p}^{\prime}\right)$ denotes the partition conjugate to $\lambda$, i.e. the partition in the $(m-p) \times p$ rectangle whose $i$-th part is equal to the length of the $i$-th column of the Young diagram of $\lambda$.

By $H_{k}$, for $m-p<k \leqslant m$, respectively $E_{k}$, for $p<k \leqslant m$, we denote the determinants from (1.4), respectively (1.3), above, corresponding to partitions $(k)$, respectively $(1)^{k}$, for the appropriate $k$ :

$$
\begin{aligned}
& H_{k}=\operatorname{det}\left(\tau^{j-1} e_{1+j-i}\right)_{1 \leqslant i, j \leqslant k}, \\
& E_{k}=\operatorname{det}\left(\tau^{1-j} h_{1+j-i}\right)_{1 \leqslant i, j \leqslant k} .
\end{aligned}
$$

With this notation, we present the main result of this paper:

Theorem 1.1. (a) There is a canonical isomorphism of $\Lambda[q]$-algebras

$$
\Lambda[q]\left[h_{1}, \ldots, h_{m-p}\right] /\left\langle E_{p+1}, \ldots, E_{m-1}, E_{m}+(-1)^{m-p} q\right\rangle \longrightarrow Q H_{T}^{*}(X),
$$

sending $h_{i}$ to $\sigma_{(i)}$. More generally, the image of $s_{\lambda}$ is the Schubert class $\sigma_{\lambda}$.

(b) (Dual version) There is a canonical isomorphism of $\Lambda[q]$-algebras

$$
\Lambda[q]\left[e_{1}, \ldots, e_{p}\right] /\left\langle H_{m-p+1}, \ldots, H_{m-1}, H_{m}+(-1)^{p} q\right\rangle \longrightarrow Q H_{T}^{*}(X),
$$

sending $e_{j}$ to $\sigma_{(1)^{j}}$ and $\widetilde{s}_{\lambda}$ to the Schubert class $\sigma_{\lambda}$.

Presentations by generators and relations for the equivariant quantum cohomology of the type A complete flag manifolds have also been obtained by A. Givental and B. Kim in [13]. Their approach was later used by A. Astashkevich and V. Sadov in [2] and independently by B. Kim in [15] to compute such presentations for the partial flag manifolds. In general, for homogeneous spaces $X=G / B$ ( $G$ a connected, semisimple, complex Lie group and $B$ a Borel subgroup) this was done by B. Kim in [17]. In the case of the Grassmannian, Kim's presentation from [15] is given as

$$
\Lambda\left[a_{1}, \ldots, a_{p}, b_{1}, \ldots, b_{m-p}\right] / I
$$

where $I$ is the ideal generated by $\sum_{i+j=k} a_{i} b_{j}=e_{k}\left(T_{1}, \ldots, T_{m}\right)$ and $a_{p} b_{m-p}=$ $e_{m}\left(T_{1}, \ldots, T_{m}\right)+(-1)^{p} q$; the sum is over integers $i, j$ such that $0 \leqslant i \leqslant p$ and 
$0 \leqslant j \leqslant m-p ; k$ varies between 1 and $m-1$ and $a_{0}=b_{0}=1$; and $e_{k}$ denotes the elementary symmetric function. We also note the results from [23], where a recursive relation, derived from a multiplication rule with the class $\sigma_{(1)}$ (a PieriChevalley rule), gives another method to compute the EQLR coefficients.

1.2. Idea of proof. The proof of the theorem uses the theory of factorial Schur functions and a characterization of the equivariant quantum cohomology ([23], cf. Proposition 3.2 below). We first prove the "dual version" of the statement. For that we show an equivalent result, where in all the formulae $\tau^{s} e_{j}$ is replaced by $e_{j}\left(x \mid \tau^{s} t\right)$ (here $x=\left(x_{1}, \ldots, x_{p}\right)$ and $t=\left(t_{i}\right)$ is the sequence defined in the beginning of $\$ 1.1)$. A key role in this "translation" is played by a factorial version of the Jacobi-Trudi formula (cf. [22], p. 56). Then we prove that the images of the polynomials $s_{\lambda}(x \mid t)$, for $\lambda$ included in the $p \times(m-p)$ rectangle, form a $\Lambda[q]$-basis in the claimed presentation. A special multiplication formula, due to Molev and Sagan ([26]; see also [18]), computes the product $s_{\lambda}(x \mid t) s_{(1)}(x \mid t)$ as a sum of $s_{\mu}(x \mid t)$, but with $\mu$ having possibly a larger part than $m-p$. Again using the factorial Jacobi-Trudi formula, we prove that, modulo the relations ideal, this multiplication is precisely the equivariant quantum Pieri-Chevalley rule (see [23]). But this rule completely determines $Q H_{T}^{*}(X)$, and so the "dual" statement is proved. To prove the first statement, we construct a morphism from $\Lambda[q]\left[h_{1}, \ldots, h_{m-p}\right]$ to the dual presentation and show that its kernel is the claimed ideal of relations.

\section{FACTORIAL Schur FUnCtions}

This section presents those properties of the factorial Schur functions which are used later in the paper. The factorial Schur function $s_{\lambda}(x \mid a)$ is a homogeneous polynomial in two sets of variables: $x=\left(x_{1}, \ldots, x_{p}\right)$ and a doubly infinite sequence $a=\left(a_{i}\right)_{i \in \mathbb{Z}}$. An initial (nonhomogeneous) version of these polynomials, for $a_{i}=i-1$ if $1 \leqslant i \leqslant p$ and 0 otherwise, was first studied by L. Biedenharn and J. Louck in [5], then by W. Chen and J. Louck in [9]. The general version was considered by I. Macdonald [21], then studied further in [14, 25, 26] and [22], Ch. I.3. These functions, as well as a different version of them, called shifted Schur functions (see $[27,28]$ ), play an important role in the study of the center of the universal enveloping algebra of $\mathfrak{g l}(n)$, Capelli identities and quantum immanants. In a geometric context, the factorial Schur functions appeared in [18], expressing the result of the localization of an equivariant Schubert class to a $T$-fixed point.

For any variable $y$ and any sequence $\left(a_{i}\right)$ define the "generalized factorial power":

$$
(y \mid a)^{k}=\left(y-a_{1}\right) \cdots\left(y-a_{k}\right) .
$$

Let $\lambda$ be a partition with at most $p$ parts. Following [26], define the factorial Schur function $s_{\lambda}(x \mid a)$ to be

$$
s_{\lambda}(x \mid a)=\frac{\operatorname{det}\left[\left(x_{j} \mid a\right)^{\lambda_{i}+p-i}\right]_{1 \leqslant i, j \leqslant p}}{\operatorname{det}\left[\left(x_{j} \mid a\right)^{p-i}\right]_{1 \leqslant i, j \leqslant p}} .
$$

Denote by $h_{k}(x \mid a)$ (respectively $e_{k}(x \mid a)$ ) the factorial complete homogeneous Schur functions (respectively the factorial elementary Schur functions). We adopt the usual convention that $h_{k}(x \mid a)$ and $e_{k}(x \mid a)$ are equal to zero if $k$ is negative. For $k$ larger than $p, e_{k}(x \mid a)$ is set equal to zero, also by convention. Let $\mathbb{Z}[a]$ denote the ring of polynomials in variables $a_{i}$ (i-integer). We start enumerating the relevant 
properties of the factorial Schur functions:

(A) (Basis.) The factorial Schur functions $s_{\lambda}(x \mid a)$, where $\lambda$ has at most $p$ parts, form a $\mathbb{Z}[a]$-basis for the ring of polynomials in $\mathbb{Z}[a][x]$ symmetric in the $x$-variables ([22], I.3, p. 55). This is a consequence of the fact that

$$
s_{\lambda}(x \mid a)=s_{\lambda}(x)+\text { terms of lower degree in } x .
$$

Then

$$
s_{\lambda}(x \mid a) s_{\mu}(x \mid a)=\sum_{\nu} c_{\lambda \mu}^{\nu}(a) s_{\nu}(x \mid a)
$$

where the coefficients $c_{\lambda \mu}^{\nu}(a)$ are homogeneous polynomials in $\mathbb{Z}[a]$ of degree $|\lambda|+$ $|\mu|-|\nu|$. They are equal to the usual Littlewood-Richardson coefficients $c_{\lambda \mu}^{\nu}$ if $|\lambda|+|\mu|=|\nu|$ and are equal to 0 if $|\lambda|+|\mu|<|\nu|$ (cf. [26], §2).

(B) (Vanishing Theorem, [26], Thm. 2.1, see also [27].) Let $\lambda=\left(\lambda_{1}, \ldots, \lambda_{p}\right)$ and $\rho=\left(\rho_{1}, \ldots, \rho_{p}\right)$ be two partitions of length at most $p$. Define the sequence $a_{\rho}$ by $a_{\rho}=\left(a_{\rho_{1}+p}, \ldots, a_{\rho_{p}+1}\right)$. Then

$$
s_{\lambda}\left(a_{\rho} \mid a\right)= \begin{cases}0 & \text { if } \lambda \subsetneq \rho, \\ \prod_{(i, j) \in \lambda}\left(a_{\lambda_{i}+p-i+1}-a_{p-\lambda_{j}^{\prime}+j}\right) & \text { if } \lambda=\rho\end{cases}
$$

where $\lambda^{\prime}$ is the conjugate partition of $\lambda$, and $(i, j) \in \lambda$ means that $j$ varies between 1 and $\lambda_{i}$, if $\lambda_{i}>0$. We recall a consequence of the Vanishing Theorem:

Corollary 2.1. The coefficients $c_{\lambda \mu}^{\nu}(a)$ satisfy the following properties:

(i) $c_{\lambda \mu}^{\nu}(a)=0$ if $\lambda$ or $\mu$ are not included in $\nu$.

(ii) If the partitions $\mu$ and $\nu$ are equal, then

$$
c_{\lambda \mu}^{\mu}(a)=s_{\lambda}\left(a_{\mu} \mid a\right) .
$$

Proof. (i) is part of Theorem 3.1 in [26], while (ii) follows from the proof of expression (10) in [26].

(C) (Factorial Pieri-Chevalley rule; see the proof of Prop. 3.2 in [26] or [28], Thm. 9.1.) Let (1) denote the partition $(1,0, \ldots, 0)$ ( $p$ parts) and let $\lambda$ be a partition with at most $p$ parts. Then

$$
s_{(1)}(x \mid a) s_{\lambda}(x \mid a)=\sum_{\mu \rightarrow \lambda} s_{\mu}(x \mid a)+c_{(1), \lambda}^{\lambda}(a) s_{\lambda}(x \mid a)
$$

where $\mu \rightarrow \lambda$ means that $\mu$ contains $\lambda$ and has one more box than $\lambda$ (recall that, by definition, $\mu$ has at most $p$ parts). By Corollary 2.1, $c_{(1), \lambda}^{\lambda}(a)=s_{(1)}\left(a_{\lambda} \mid a\right)$ and the last expression turns out to be

$$
s_{(1)}\left(a_{\lambda} \mid a\right)=\sum_{i=1}^{p} a_{\lambda_{i}+p+1-i}-\sum_{j=1}^{p} a_{j} .
$$

(D) (Jacobi-Trudi identities; see [22], I.3, Ex. 20(c), p. 56 or [25], Thm. 3.1.) Let $\tau^{r} a$ be the sequence whose $n$-th term is $a_{n+r}$ and let $\lambda$ be a partition with at 
most $p$ parts. Then

$$
\begin{aligned}
s_{\lambda}(x \mid a) & =\operatorname{det}\left[h_{\lambda_{i}-i+j}\left(x \mid \tau^{1-j} a\right)\right]_{1 \leqslant i, j \leqslant p} \\
& =\operatorname{det}\left[e_{\lambda_{i}^{\prime}-i+j}\left(x \mid \tau^{j-1} a\right)\right]_{1 \leqslant i, j \leqslant m-p},
\end{aligned}
$$

where $\lambda^{\prime}$ is the partition conjugate of $\lambda$.

The following proposition gives an inductive way of computing the "shifted" polynomials $h_{k}\left(x \mid \tau^{s} a\right)$, respectively $e_{k}\left(x \mid \tau^{s} a\right)$, starting from the "unshifted" ones.

Proposition 2.2. The following identities hold in $\mathbb{Z}[a][x]$ :

$$
\begin{aligned}
& h_{i+1}\left(x \mid \tau^{-1} a\right)=h_{i+1}(x \mid a)+\left(a_{i+p}-a_{0}\right) h_{i}(x \mid a), \\
& e_{j+1}(x \mid \tau a)=e_{j+1}(x \mid a)+\left(a_{1}-a_{p-j+1}\right) e_{j}(x \mid a) .
\end{aligned}
$$

Proof. One uses the formulae

$$
\begin{aligned}
h_{k}(x \mid a) & =\sum_{1 \leqslant i_{1} \leqslant \ldots \leqslant i_{k} \leqslant p}\left(x_{i_{1}}-a_{i_{1}}\right)\left(x_{i_{2}}-a_{i_{2}+1}\right) \cdot \ldots \cdot\left(x_{i_{k}}-a_{i_{k}+k-1}\right), \\
e_{k}(x \mid a) & =\sum_{1 \leqslant i_{1}<\ldots<i_{k} \leqslant p}\left(x_{i_{1}}-a_{i_{1}}\right)\left(x_{i_{2}}-a_{i_{2}-1}\right) \cdot \ldots \cdot\left(x_{i_{k}}-a_{i_{k}+k-1}\right)
\end{aligned}
$$

(cf. [25], eqs. (1.2) and (1.3)). Then the computations are straightforward.

By the Jacobi-Trudi formula, $h_{i}\left(x \mid \tau^{-s} a\right)$ (respectively $e_{j}\left(x \mid \tau^{s} a\right)$ ) generate the algebra of polynomials in $\mathbb{Z}[a][x]$ symmetric in the $x$-variables. Then Proposition 2.2 implies:

Corollary 2.3. The factorial complete homogeneous (respectively elementary) symmetric functions $h_{i}(x \mid a)$ for $1 \leqslant i$ (respectively $e_{j}(x \mid a)$ for $1 \leqslant j \leqslant p$ ) generate the algebra of polynomials in $\mathbb{Z}[a][x]$ symmetric in the $x$-variables.

We will need the fact that the Jacobi-Trudi formula (2.5) generalizes to the case when $\lambda=(1)^{k}$ with $k>p$ (when, by convention, $e_{k}(x \mid a)=0$ ), and this is the content of the next proposition (see also equation (6.10) in [21]):

Proposition 2.4. The following holds for any positive integer $k>p$ :

$$
\operatorname{det}\left(h_{1+j-i}\left(x \mid \tau^{1-j} a\right)\right)_{1 \leqslant i, j \leqslant k}=0 .
$$

Proof. Denote by $E_{k}(x \mid a)$ the determinant in question. Note that, if $k \leqslant p$, this is equal to $e_{k}(x \mid a)$, by the Jacobi-Trudi formula. We will need a formula proved in [22], I.3, p. 56, Ex. 20(b):

$$
\sum_{r=0}^{p}(-1)^{r} e_{r}(x \mid a) h_{s-r}\left(x \mid \tau^{1-s} a\right)=0
$$

for any positive integer $s$. To prove the proposition, we use induction on $k \geqslant p+1$. Expanding $E_{p+1}(x \mid a)$ after the last column yields:

$$
\begin{aligned}
E_{p+1}(x \mid a) & =\sum_{r=0}^{p}(-1)^{r+1+p+1} h_{p+1-r}\left(x \mid \tau^{-p} a\right) e_{r}(x \mid a) \\
& =(-1)^{p+2} \sum_{r=0}^{p}(-1)^{r} h_{p+1-r}\left(x \mid \tau^{-p} a\right) e_{r}(x \mid a) \\
& =0
\end{aligned}
$$


where the last equality follows from (2.10) by taking $s=p+1$. Assume that $E_{k}(x \mid a)=0$ for all $p<k<k_{0}$. Expanding the determinant defining $E_{k_{0}}(x \mid t)$ after the last column, and using the induction hypothesis, yields

$$
\begin{aligned}
E_{k_{0}}(x \mid a) & =\sum_{r=0}^{p}(-1)^{r+1+k_{0}} h_{k_{0}-r}\left(x \mid \tau^{1-k_{0}} a\right) e_{r}(x \mid a) \\
& =(-1)^{k_{0}+1} \sum_{r=0}^{p}(-1)^{r} h_{k_{0}-r}\left(x \mid \tau^{1-k_{0}} a\right) e_{r}(x \mid a) \\
& =0
\end{aligned}
$$

again using (2.10) with $s=k_{0}$.

\section{Equivariant quantum cohomology of the Grassmannian}

In this section we recall some basic properties of the equivariant quantum cohomology. As before, $X$ denotes the Grassmannian $\operatorname{Gr}(p, m)$, and $\Lambda$ the polynomial ring $\mathbb{Z}\left[T_{1}, \ldots, T_{m}\right]$. The $(T-)$ equivariant quantum cohomology of the Grassmannian, denoted by $Q H_{T}^{*}(X)$, is a deformation of both equivariant and quantum cohomology rings (for details on the latter cohomologies, see e.g. [23]). More precisely, $Q H_{T}^{*}(X)$ is a graded, commutative, $\Lambda[q]$-algebra, where the degree of $q$ is equal to $m$, which has a $\Lambda[q]$-basis $\left\{\sigma_{\lambda}\right\}$ indexed by the partitions $\lambda$ included in the $p \times(m-p)$ rectangle. If $\lambda=\left(\lambda_{1}, \ldots, \lambda_{p}\right)$, the degree of $\sigma_{\lambda}$ is equal to $|\lambda|=\lambda_{1}+\ldots+\lambda_{p}$. The multiplication of two basis elements $\sigma_{\lambda}$ and $\sigma_{\mu}$ is given by the equivariant quantum Littlewood-Richardson (EQLR) coefficients $c_{\lambda, \mu}^{\nu, d}$, where $d$ is a nonnegative integer:

$$
\sigma_{\lambda} \circ \sigma_{\mu}=\sum_{d} \sum_{\nu} q^{d} c_{\lambda, \mu}^{\nu, d} \sigma_{\nu}
$$

Recall that the EQLR coefficient $c_{\lambda, \mu}^{\nu, d}$ is a homogeneous polynomial in $\Lambda$ of polynomial degree $|\lambda|+|\mu|-|\nu|-m d$. If $d=0$ one recovers the structure constant $c_{\lambda, \mu}^{\nu}$ in the equivariant cohomology of $X$, and if the polynomial degree is equal to 0 (i.e. if $|\lambda|+|\mu|=|\nu|+m d)$ the EQLR coefficient is equal to the ordinary 3-pointed, genus 0 , Gromov-Witten invariant $c_{\lambda, \mu}^{\nu, d}$. The latter is a nonnegative integer equal to the number of rational curves in $X$ passing through general translates of the Schubert varieties in $X$ corresponding to the partitions $\lambda, \mu$ and the dual of $\nu$.

The geometric definition of these coefficients can be found in [23]. In fact, for the purpose of this paper, the algebraic characterization of the equivariant quantum cohomology from Proposition 3.2 below (which has a geometric proof in loc. cit.) suffices. We only remark that the equivariant quantum Schubert classes $\sigma_{\lambda}$ are determined by the equivariant Schubert classes $\sigma_{\lambda}^{T}$, determined in turn by the Schubert varieties in $X$ defined with respect to the standard flag. ${ }^{2}$ The precise definition of $\sigma_{\lambda}^{T}$ is not presently needed, but it is given in $\S 5$, where the equivariant cohomology ring is discussed in more detail.

\footnotetext{
${ }^{2}$ Unlike the case of classical cohomology, in equivariant cohomology the Schubert class determined by a Schubert variety $\Omega_{\lambda}\left(F_{\bullet}\right)$, where $F_{\bullet}$ is a fixed flag in $\mathbb{C}^{m}$, depends on $F_{\bullet}$.
} 
From now on we specialize the sequence $a=\left(a_{i}\right)_{i \in \mathbb{Z}}$ from the previous section to one, denoted $t=\left(t_{i}\right)_{i \in \mathbb{Z}}$, encoding the equivariant parameters $T_{i}$ :

$$
t_{i}= \begin{cases}T_{m-i+1} & \text { if } 1 \leqslant i \leqslant m \\ 0 & \text { otherwise }\end{cases}
$$

Using this sequence, we next recall the equivariant quantum Pieri-Chevalley rule, as proved in [23]. Given a partition $\lambda$, we denote by $\lambda^{-}$the (uniquely determined) partition obtained by removing $m-1$ boxes from the border rim of $\lambda$ (recall that the border rim of a Young diagram is the set of boxes that intersect the diagram's SE border; see also the figure below).

$\lambda$

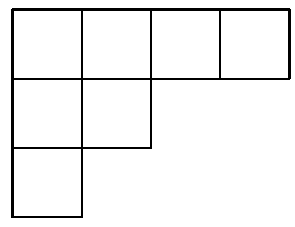

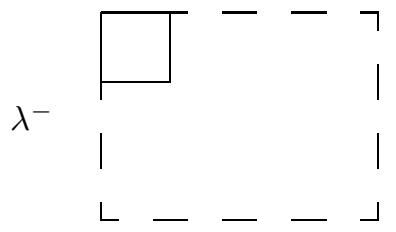

Figure 1. Example: $p=3, m=7, \lambda=(4,2,1) ; \lambda^{-}=(1)$.

If $\lambda=\left(\lambda_{1}, \ldots, \lambda_{p}\right)$, note that $\lambda^{-}$exists only if $\lambda_{1}=m-p$ and $\lambda_{p}>0$.

Proposition 3.1 (Equivariant quantum Pieri-Chevalley rule; cf. [23], Thm. 1). The following formula holds in $Q H_{T}^{*}(X)$ :

$$
\sigma_{\lambda} \circ \sigma_{(1)}=\sum_{\mu \rightarrow \lambda} \sigma_{\mu}+c_{\lambda,(1)}^{\lambda}(t) \sigma_{\lambda}+q \sigma_{\lambda^{-}}
$$

where, by the formula (2.3), $c_{\lambda,(1)}^{\lambda}(t)$ is equal to

$$
c_{\lambda,(1)}^{\lambda}(t)=\sum_{i=1}^{p} T_{m-p+i-\lambda_{i}}-\sum_{j=m-p+1}^{m} T_{j} .
$$

The last term is omitted if $\lambda^{-}$does not exist.

It turns out that the equivariant quantum Pieri-Chevalley rule completely determines the equivariant quantum cohomology algebra, in the following sense:

Proposition 3.2 ([23], Corollary 7.1). Let $(A, \diamond)$ be a graded, commutative, associative $\Lambda[q]$-algebra with unit such that:

1. A has an additive $\Lambda[q]$-basis $\left\{s_{\lambda}\right\}$ (graded as usual).

2. The equivariant quantum Pieri-Chevalley rule holds, i.e.

$$
s_{\lambda} \diamond s_{(1)}=\sum_{\mu \rightarrow \lambda} s_{\mu}+c_{\lambda,(1)}^{\lambda}(t) s_{\lambda}+q s_{\lambda^{-}}
$$

where the last term is omitted if $\lambda^{-}$does not exist.

Then there is a canonical isomorphism of the $\Lambda[q]$-algebras A and $Q H_{T}^{*}(G r(p, m))$, sending $s_{\lambda}$ to the equivariant quantum Schubert class $\sigma_{\lambda}$.

This proposition will be the main tool in proving the presentation and equivariant quantum Giambelli formula from the next section. 


\section{Proof of the theorem}

The strategy for the proof is to "guess" candidates for the presentation and for the polynomial representatives, using the insight provided by the similar results in quantum cohomology (see e.g. [4]) and some related results in equivariant cohomology $([18], \S 6)$. Then one attempts to prove that the guessed polynomials form a $\Lambda[q]$-basis in the candidate presentation, and they multiply according to the EQ Pieri-Chevalley rule. Proposition 3.2 will ensure that the guessed algebra will be canonically isomorphic to $Q H_{T}^{*}(X)$ and that the polynomials considered will represent the equivariant quantum Schubert classes.

It turns out that each of the quantum presentations from [4] (the usual one, involving the $h$ variables, and the "dual" one, involving the variables $e$ ) implies an equivariant quantum presentation (see respectively Theorems 4.3 and 4.2 below). The equivariant generalizations are obtained by taking the factorial versions, via the factorial Jacobi-Trudi formula ( $\$ 2$, property D), of all the expressions involved in the original quantum presentations.

Before stating the first result, we recall the notation from the introduction: $h_{1}, \ldots, h_{m-p}$ and $e_{1}, \ldots, e_{p}$ denote two sets of indeterminates; the definitions of $\tau^{-s} h_{i}$, $\tau^{s} e_{j}$ and of $H_{k}(m-p<k \leqslant m)$, respectively $E_{k}(p<k \leqslant m)$, are those given in the equations (1.1), (1.2) and (1.5), (1.6) above. For $\lambda$ in the $p \times(m-p)$ rectangle recall that:

$$
s_{\lambda}=\operatorname{det}\left(\tau^{1-j} h_{\lambda_{i}+j-i}\right)_{1 \leqslant i, j \leqslant p}
$$

respectively

$$
\widetilde{s}_{\lambda}=\operatorname{det}\left(\tau^{j-1} e_{\lambda_{i}^{\prime}+j-i}\right)_{1 \leqslant i, j \leqslant m-p}
$$

(cf. (1.3) and (1.4)) with the usual conventions that $h_{k}=0$ for $k<0$ and $k>m-p$, respectively $e_{i}=0$, if $i<0$ or $i>p$. Before proving the theorem, we need a Nakayama-type result, which will be used several times in the paper:

Lemma 4.1 (cf. [10], Exerc. 4.6). Let $M$ be an $R$-algebra graded by nonnegative integers. Assume that $R$ is also graded (by nonnegative integers) and let $I$ be a homogeneous ideal in $R$ consisting of elements of positive degree. Let $m_{1}, \ldots, m_{k}$ be homogeneous elements whose images generate $M / I M$ as an $R / I$-module. Then $m_{1}, \ldots, m_{k}$ generate $M$ as an $R$-module.

Proof. Let $m$ be a nonzero homogeneous element of $M$. We use induction on its degree. Assume $\operatorname{deg} m=0$. The hypothesis implies that

$$
m=r_{1} m_{1}+\ldots+r_{k} m_{k} \bmod I M
$$

where $r_{i}$ are elements in $R$. Since $I$ contains only elements of positive degree, it follows that the equality holds in $M$ as well. Now let $\operatorname{deg} m>0$. Writing $m$ as in (4.3) implies that

$$
m-\sum_{i} r_{i} m_{i}=\sum_{j} a_{j} m_{j}^{\prime}
$$

for some (finitely many) $a_{j} \in I$ and $m_{j}^{\prime} \in M$. Again, since $I$ contains only elements of positive degree, $\operatorname{deg} m_{j}^{\prime}<\operatorname{deg} m$ for each $j$. The induction hypothesis implies that each $m_{j}^{\prime}$ is an $R$-combination of $m_{i}$ 's, which finishes the proof. 
We next prove the "dual version" statement from the main theorem.

Theorem 4.2. There exists a canonical isomorphism of $\Lambda[q]$-algebras

$$
\Lambda[q]\left[e_{1}, \ldots, e_{p}\right] /\left\langle H_{m-p+1}, \ldots, H_{m}+(-1)^{p} q\right\rangle \longrightarrow Q H_{T}^{*}(X),
$$

sending $e_{i}$ to $\sigma_{(1)^{i}}$ and $\widetilde{s}_{\lambda}$ to the equivariant quantum Schubert class $\sigma_{\lambda}$.

Proof. Note first that

$$
e_{j}(x \mid t)=e_{j}(x)+f(t, x),
$$

where $f(t, x)$ is a homogeneous polynomial in the variables $x$ and $t$, but of degree in the variables $x$ less than $j$. Since the usual elementary symmetric functions $e_{1}(x), \ldots, e_{p}(x)$ are algebraically independent over $\mathbb{Z}$, it follows that the elementary factorial Schur functions $e_{1}(x \mid t), \ldots, e_{p}(x \mid t)$ are algebraically independent over $\Lambda$. Then there is a canonical isomorphism

$$
\Lambda[q]\left[e_{1}(x \mid t), \ldots, e_{p}(x \mid t)\right] \rightarrow \Lambda[q]\left[e_{1}, \ldots, e_{p}\right],
$$

sending $s_{\lambda}(x \mid t)$ to $\widetilde{s}_{\lambda}$ and $h_{m-p+i}(x \mid t)(1 \leqslant i \leqslant p)$ to $H_{m-p+i}$, by the factorial Jacobi-Trudi identity. This induces an isomorphism between

$$
A:=\Lambda[q]\left[e_{1}(x \mid t), \ldots, e_{p}(x \mid t)\right] /\left\langle h_{m-p+1}(x \mid t), \ldots, h_{m}(x \mid t)+(-1)^{p} q\right\rangle
$$

and

$$
\Lambda[q]\left[e_{1}, \ldots, e_{p}\right] /\left\langle H_{m-p+1}, \ldots, H_{m}+(-1)^{p} q\right\rangle .
$$

By Proposition 3.2, it remains to show that the images of $s_{\lambda}(x \mid t)$ in $A$, as $\lambda$ varies over the partitions included in the $p \times(m-p)$ rectangle, form a $\Lambda[q]$-basis of $A$, satisfying the equivariant quantum Pieri-Chevalley rule.

Generating set. This follows from Lemma 4.1, applied to $M=A, R=\Lambda[q]$ and $I$ the ideal generated by $q$ and $T_{1}, \ldots, T_{p}$ (in which case $M / I$ is the classical cohomology of $X$ ).

Linear independence. Assume that $\sum q^{d_{\lambda}} c_{\lambda} s_{\lambda}(x \mid t)=0$ in $A$, for $c_{\lambda}$ in $\Lambda$, where $\lambda$ is included in the $p \times(m-p)$ rectangle. This implies that $\sum q^{d_{\lambda}} c_{\lambda} s_{\lambda}(x \mid t)$ is in the ideal generated by $h_{m-p+1}(x \mid t), \ldots, h_{m}(x \mid t)+(-1)^{p} q$. By Corollary 2.1 (i), any element of this ideal can be written as:

$$
\sum_{\mu} q^{d_{\mu}^{\prime}} c_{\mu}^{\prime} s_{\mu}(x \mid t)+\sum_{\nu} q^{d_{\nu}^{\prime}} c_{\nu}^{\prime \prime} s_{\nu}(x \mid t)\left(h_{m}(x \mid t)+(-1)^{p} q\right)
$$

where $\mu, \nu$ have at most $p$ parts, $\mu$ is outside the $p \times(m-p)$ rectangle, and $c_{\mu}^{\prime}, c_{\nu}^{\prime \prime}$ are in $\Lambda$. Note that $s_{\nu}(x \mid t) h_{m}(x \mid t)$ also expands into a sum of factorial Schur functions indexed by partitions outside the $p \times(m-p)$ rectangle. Since the factorial Schur functions form a $\Lambda[q]$-basis for the polynomials in $\Lambda[q]\left[x_{1}, \ldots, x_{p}\right]$ symmetric in the $x$-variables, it follows that all $c_{\lambda}$ (and $c_{\mu}^{\prime}, c_{\nu}^{\prime \prime}$ ) must be equal to zero, as desired.

Equivariant quantum Pieri-Chevalley. The factorial Pieri-Chevalley rule $(\S 2$, property $(\mathrm{C}))$ states that if $\lambda$ is included in the $p \times(m-p)$ rectangle, then

$$
s_{\lambda}(x \mid t) \cdot s_{(1)}(x \mid t)=\sum_{\mu} s_{\mu}(x \mid t)+c_{(1), \lambda}^{\lambda}(t) s_{\lambda}(x \mid t)+s_{\bar{\lambda}}(x \mid t),
$$

where $\mu$ runs over all partitions in the $p \times(m-p)$ obtained from $\lambda$ by adding one box; the last term is omitted if $\lambda_{1}$, the first part of $\lambda$, is not equal to $m-p$. If $\lambda_{1}=m-p$, then $\bar{\lambda}=\left(\bar{\lambda}_{1}, \ldots, \bar{\lambda}_{p}\right)$ is given by adding a box to the first row of $\lambda$, i.e. 
$\bar{\lambda}_{1}=m-p+1$ and $\bar{\lambda}_{i}=\lambda_{i}$ for $i \geqslant 2$. Since the images of $s_{\lambda}(x \mid t)$, as $\lambda$ varies in the $p \times(m-p)$ rectangle, form a $\Lambda[q]$-basis for $A$, it is enough to show that

$$
s_{\bar{\lambda}}(x \mid t)=q s_{\lambda^{-}}(x \mid t) \quad \bmod J
$$

where $J$ is the ideal generated by $h_{m-p+1}(x \mid t), \ldots, h_{m}(x \mid t)+(-1)^{p} q$. By the factorial Jacobi-Trudi formula ( $\S 2$, property $(\mathrm{D}))$, it follows that

$$
s_{\bar{\lambda}}(x \mid t)=\operatorname{det}\left(\begin{array}{cccc}
h_{m-p+1}(x \mid t) & h_{m-p+2}\left(x \mid \tau^{-1} t\right) & \ldots & h_{m}\left(x \mid \tau^{1-p} t\right) \\
h_{\lambda_{2}-1}(x \mid t) & h_{\lambda_{2}}\left(x \mid \tau^{-1} t\right) & \ldots & h_{\lambda_{2}+p-2}\left(x \mid \tau^{1-p} t\right) \\
\vdots & \vdots & \vdots & \vdots \\
\ldots & \ldots & \ldots & h_{\lambda_{p}}\left(x \mid \tau^{1-p} t\right)
\end{array}\right)
$$

We next analyze the first row of this determinant.

Claim. Let $i, j$ be two integers such that $2 \leqslant j \leqslant i \leqslant p$. Then

$$
h_{m-p+i}\left(x \mid \tau^{1-j} t\right)=h_{m-p+i}\left(x \mid \tau^{1-(j-1)} t\right) \text {. }
$$

Proof of the Claim. Equation (2.6) implies that

$$
h_{j}\left(x \mid \tau^{-s} t\right)=h_{j}\left(x \mid \tau^{-s+1} t\right)+\left(t_{j+p-s}-t_{-s+1}\right) h_{j-1}\left(x \mid \tau^{-s+1} t\right),
$$

hence,

$h_{m-p+i}\left(x \mid \tau^{1-j} t\right)=h_{m-p+i}\left(x \mid \tau^{1-(j-1)} t\right)+\left(t_{m+1+i-j}-t_{2-j}\right) h_{m-p+i-1}\left(x \mid \tau^{1-(j-1)} t\right)$.

The Claim then follows from the definition of $\left(t_{i}\right)$, since $t_{m+1+i-j}=t_{2-j}=0$.

It follows that for any integer $1 \leqslant i \leqslant p$,

$$
h_{m-p+i}\left(x \mid \tau^{1-i} t\right)=h_{m-p+i}(x \mid t) .
$$

In particular,

$$
h_{m-p+i}\left(x \mid \tau^{1-i} t\right)=0 \quad \bmod J
$$

if $1 \leqslant i \leqslant p-1$, and

$$
h_{m}\left(x \mid \tau^{1-p} t\right)=(-1)^{p+1} q \bmod J .
$$

Therefore, expanding the determinant in (4.4) after the first row, yields:

$$
s_{\bar{\lambda}}(x \mid t)=(-1)^{p+1}(-1)^{p+1} q \operatorname{det}\left(\begin{array}{cccc}
h_{\lambda_{2}-1}(x \mid t) & h_{\lambda_{2}}\left(x \mid \tau^{-1} t\right) & \ldots & h_{\lambda_{2}+p-3}\left(x \mid \tau^{2-p} t\right) \\
\vdots & \vdots & \vdots & \vdots \\
\ldots & \ldots & \ldots & h_{\lambda_{p}-1}\left(x \mid \tau^{2-p} t\right)
\end{array}\right)
$$

in $A$. If $\lambda_{p}=0$, the last row of the determinant in (4.6) contains only zeroes; if $\lambda_{p}>$ 0 , the determinant is equal to $s_{\lambda^{-}}(x \mid t)$, by the Jacobi-Trudi formula. Summarizing, $s_{\bar{\lambda}}(x \mid t)$ is equal to $q s_{\lambda^{-}}(x \mid t)$ in $A$, or it is equal to zero if $\lambda^{-}$does not exist. This finishes the proof of the equivariant quantum Pieri-Chevalley rule, hence also the proof of the theorem.

We are ready to prove the first part of the main result, which involves the $h$ variables. We use the notation preceding Theorem 4.2 above.

Theorem 4.3. There exists a canonical isomorphism of $\Lambda[q]$-algebras

$$
\Lambda[q]\left[h_{1}, \ldots, h_{m-p}\right] /\left\langle E_{p+1}, \ldots, E_{m}+(-1)^{m-p} q\right\rangle \longrightarrow Q H_{T}^{*}(X),
$$

such that $h_{j}$ is sent to $\sigma_{(j)}$ and $s_{\lambda}$ to the equivariant quantum Schubert class $\sigma_{\lambda}$. 
Proof. Consider the $\Lambda[q]$-algebra morphism

$\Psi: \Lambda[q]\left[h_{1}, \ldots, h_{m-p}\right] \rightarrow \Lambda[q]\left[e_{1}(x \mid t), \ldots, e_{p}(x \mid t)\right] /\left\langle h_{m-p+1}(x \mid t), \ldots, h_{m}(x \mid t)+(-1)^{p} q\right\rangle$ sending $h_{k}$ to the image of $h_{k}(x \mid t)=\operatorname{det}\left(e_{1+j-i}\left(x \mid \tau^{1-j} t\right)\right)_{1 \leqslant i, j \leqslant k}$. Recall that the last quotient is denoted by $A$ and it is canonically isomorphic to $Q H_{T}^{*}(X)$, by the previous proof. We will show that the images under $\Psi$ of $E_{p+1}, E_{p+2}, \ldots, E_{m-1}, E_{m}+$ $(-1)^{m-p} q$ are equal to zero in $A$ (where $E_{i}$ is defined by equation (1.6)). First, we need the following Claim:

Claim. The following formulae hold in $A$ :

$$
\Psi\left(\tau^{-s} h_{j}\right)=h_{j}\left(x \mid \tau^{-s} t\right),
$$

for any nonnegative integers $s$ and $j$ with $j<m$, and

$$
\Psi\left(\tau^{-(m-1)} h_{m}\right)=h_{m}\left(x \mid \tau^{-(m-1)} t\right)+(-1)^{p} q .
$$

Proof of the Claim. By definition, both $\tau^{-s} h_{j}$ and $h_{j}\left(x \mid \tau^{-s} t\right)$ satisfy the same recurrence relations (given respectively by the equations (1.1) and (2.6)). This implies that there exist polynomials $P_{1}(t), \ldots, P_{s}(t)$ in $\Lambda$, with $\operatorname{deg} P_{k}(t)=k$, such that

$$
\tau^{-s} h_{j}=h_{j}+\sum_{k=1}^{s} P_{k}(t) h_{j-k},
$$

respectively

$$
h_{j}\left(x \mid \tau^{-s} t\right)=h_{j}(x \mid t)+\sum_{k=1}^{s} P_{k}(t) h_{j-k}(x \mid t) .
$$

If $j \leqslant m-p$, then $\Psi\left(h_{j}\right)=h_{j}(x \mid t)$ in $A$, by the definition of $\Psi$, thus

$$
\Psi\left(\tau^{-s} h_{j}\right)=h_{j}\left(x \mid \tau^{-s} t\right) .
$$

If $m-p+1 \leqslant j<m, h_{j}=0$ by convention, whereas $h_{j}(x \mid t)=0$ in $A$, so equation (4.10) also holds in this case. If $s=m-1$ and $j=m$, we have

$$
\begin{aligned}
\Psi\left(\tau^{-(m-1)} h_{m}\right) & =\Psi\left(h_{m}+\sum_{k=1}^{m-1} P_{k}(t) h_{m-k}\right) \\
& =\Psi\left(h_{m}\right)+\sum_{k=1}^{m-1} P_{k}(t) \Psi\left(h_{m-k}\right) \\
& =h_{m}(x \mid t)+(-1)^{p} q+\sum_{k=1}^{m-1} P_{k}(t) h_{m-k}(x \mid t) \\
& =h_{m}\left(x \mid \tau^{-(m-1)} t\right)+(-1)^{p} q,
\end{aligned}
$$

where the third equality follows from the fact that $h_{m}=0$ and $h_{m}(x \mid t)+(-1)^{p} q=0$ in $A$; the fourth equality follows from the expansion (4.9) of $h_{m}\left(x \mid \tau^{-(m-1)} t\right)$.

By definition, $\Psi\left(E_{i}\right)$ is equal to the image in $A$, through $\Psi$, of

$$
\operatorname{det}\left(\begin{array}{cccccc}
h_{1} & \tau^{-1} h_{2} & \ldots & \tau^{-(s-1)} h_{s} & \ldots & \tau^{-(i-1)} h_{i} \\
1 & \tau^{-1} h_{1} & \ldots & \ldots & \ldots & \tau^{-(i-1)} h_{i-1} \\
\vdots & \vdots & \vdots & \vdots & \vdots & \vdots \\
0 & \ldots & \ldots & 0 & 1 & \tau^{-(i-1)} h_{1}
\end{array}\right)
$$


If $p+1 \leqslant i<m$, this determinant contains only $\tau^{-s} h_{j}$ with $j<m$. Then, by equation (4.7) from the Claim, $\Psi\left(E_{i}\right)$ is the image in $A$ of the determinant

$$
\operatorname{det}\left(\begin{array}{cccccc}
h_{1}(x \mid t) & h_{2}\left(x \mid \tau^{-1} t\right) & \ldots & h_{s}\left(x \mid \tau^{-(s-1)} t\right) & \ldots & h_{i}\left(x \mid \tau^{-(i-1)} t\right) \\
1 & h_{1}\left(x \mid \tau^{-1} t\right) & \ldots & \ldots & \ldots & h_{i-1}\left(x \mid \tau^{-(i-1)} t\right) \\
\vdots & \vdots & \vdots & \vdots & \vdots & \vdots \\
0 & \ldots & \ldots & 0 & 1 & h_{1}\left(x \mid \tau^{-(i-1)} t\right)
\end{array}\right)
$$

which, by Proposition 2.4, is equal to zero, since $i>p$. To compute the image of $\Psi\left(E_{m}\right)$ we use both equations (4.7) and (4.8). Then $\Psi\left(E_{m}\right)$ is equal to the image in $A$ of

$$
\begin{aligned}
& \operatorname{det}\left(\begin{array}{cccccc}
h_{1}(x \mid t) & h_{2}\left(x \mid \tau^{-1} t\right) & \ldots & h_{s}\left(x \mid \tau^{-(s-1)} t\right) & \ldots & h_{m}\left(x \mid \tau^{-(m-1)} t\right) \\
\vdots & \vdots & \vdots & \vdots & \vdots & \vdots \\
0 & \ldots & \ldots & 0 & 1 & h_{1}\left(x \mid \tau^{-(m-1)} t\right)
\end{array}\right) \\
& +\operatorname{det}\left(\begin{array}{ccccc}
0 & 0 & \ldots & 0 & (-1)^{p} q \\
1 & h_{1}\left(x \mid \tau^{-1} t\right) & \ldots & \ldots & h_{m-1}\left(x \mid \tau^{-(m-1)} t\right) \\
\vdots & \vdots & \vdots & \vdots & \vdots \\
0 & \ldots & 0 & 1 & h_{1}\left(x \mid \tau^{-(m-1)} t\right)
\end{array}\right)
\end{aligned}
$$

The first determinant is equal to zero, by Proposition 2.4, and the second is $(-1)^{m+1} \cdot(-1)^{p} q$. It follows that $\Psi\left(E_{m}\right)+(-1)^{m-p} q$ is equal to zero in $A$, as claimed. Thus $\Psi$ induces a $\Lambda[q]$-algebra morphism

$$
\Psi^{\prime}: \Lambda[q]\left[h_{1}, \ldots, h_{m-p}\right] /\left\langle E_{p+1}, \ldots, E_{m}+(-1)^{m-p} q\right\rangle \longrightarrow A .
$$

Note that $\tau^{-s} h_{m}$ does not appear in the determinant defining $s_{\lambda}$, for any $\lambda$ in the $p \times(m-p)$ rectangle, so $\Psi^{\prime}$ sends $s_{\lambda}$ to the image of $s_{\lambda}(x \mid t)$ in $A$. Applying Lemma 4.1 with $M=\Lambda[q]\left[h_{1}, \ldots, h_{m-p}\right] /\left\langle E_{p+1}, \ldots, E_{m}+(-1)^{m-p} q\right\rangle$ and $I$, the ideal generated by $q$ and $T_{1}, \ldots, T_{m}$ implies that the polynomials $s_{\lambda}$ generate $\Lambda[q]\left[h_{1}, \ldots, h_{m-p}\right] /\left\langle E_{p+1}, \ldots ., E_{m}+(-1)^{m-p} q\right\rangle$ as a $\Lambda[q]$-module. Since their images through $\Psi^{\prime}$ form a $\Lambda[q]$-basis, they must form a $\Lambda[q]$-basis, as well. Hence $\Psi^{\prime}$ is an isomorphism, as desired.

Remarks. 1. Theorems 4.2 and 4.3 are proved without using the corresponding results from quantum cohomology. In particular, we obtain a new proof for Bertram's quantum Giambelli formula (see [3]). (Recall that the quantum cohomology ring of $X$ is a graded $\mathbb{Z}[q]$-algebra isomorphic to $Q H_{T}^{*}(X) /\left(T_{1}, \ldots, T_{m}\right)$, hence the quantum Giambelli formula is obtained by taking $T_{1}=\ldots=T_{m}=0$ in the determinants from the above-mentioned theorems.) In fact, Bertram's proof is purely geometrical; it uses a careful analysis of a certain Quot scheme and the explicit enumerative interpretation of the (3-pointed, genus 0) Gromov-Witten invariants. Unfortunately, the equivariant version of the Gromov-Witten invariants does not have an explicit enumerative interpretation. It would be very interesting to compute these invariants via the (equivariant) intersection theory of the Quot scheme, therefore extending Bertram's approach to the equivariant setting.

2. A "moduli-free" proof of the quantum Giambelli formula was later obtained by A. Buch in [8], as a consequence of the quantum Pieri formula (i.e. a rule to multiply a Schubert class $\sigma_{\lambda}$ by a generator $\left.\sigma_{(i)}\right)$. It uses the notions of kernel and span of a rational curve to $X$. These notions have also been used in the (geometric) 
proof of the equivariant quantum Pieri-Chevalley formula ([23], cf. Proposition 3.1 above), which involves the equivariant cohomology of a moduli space of stable maps to $X$. For other (combinatorial) proofs of the quantum Giambelli formula see $[4,29]$.

\section{Giambelli formulae in EQuivariant cohomology}

The goal of this section is to state the equivariant Giambelli formulae implied by their equivariant quantum counterparts from the previous section. We will also use this opportunity to rigourously define the equivariant Schubert classes involved, and provide, without proof, a geometric interpretation for the factorial Schur functions.

Let $T$ be the usual torus, and let $E T \rightarrow B T$ be the universal $T$-bundle. If $X$ is a topological space with a $T$-action, there is an induced $T$-action on $E T \times X$ given by $t \cdot(e, x)=\left(e t^{-1}, t x\right)$. The (topological) quotient space $(E T \times X) / T$ is denoted by $X_{T}$. By definition, the $\left(T-\right.$ )equivariant cohomology of $X$, denoted $H_{T}^{*}(X)$, is equal to the usual cohomology of $X_{T}$. The $X$-bundle $X_{T} \rightarrow B T$ gives $H_{T}^{*}(X)$ the structure of a $\Lambda$-algebra, where $\Lambda$ denotes the equivariant cohomology of a point $H_{T}^{*}(p t)=H^{*}(B T)$.

Now let $X$ be the Grassmannian of subspaces of dimension $p$ in $\mathbb{C}^{m}$ with the $T$-action induced from the usual $G L(m)$-action. We next define the equivariant Schubert classes which determine the equivariant quantum classes $\sigma_{\lambda}$ used in previous sections (see also [23]). Let

$$
F_{\bullet}:(0) \subset F_{1} \subset \ldots \subset F_{m}=\mathbb{C}^{m}
$$

be the standard flag, so $F_{i}=\left\langle f_{1}, \ldots, f_{i}\right\rangle$ and $f_{i}=(0, \ldots, 1, \ldots, 0)$ (with 1 in the $i$-th position). If $\lambda=\left(\lambda_{1}, \ldots, \lambda_{p}\right)$ is a partition included in the $p \times(m-p)$ rectangle, define the Schubert variety $\Omega_{\lambda}\left(F_{\bullet}\right)$ by

$$
\Omega_{\lambda}\left(F_{\bullet}\right)=\left\{V \in X: \operatorname{dim} V \cap F_{m-p+i-\lambda_{i}} \geqslant i\right\} .
$$

Since the flag $F_{\bullet}$ is $T$-invariant, the Schubert variety $\Omega_{\lambda}\left(F_{\bullet}\right)$ will be $T$-invariant as well, so it determines a Schubert class $\sigma_{\lambda}^{T}$ in $H_{T}^{2|\lambda|}(X)$. The following result is a consequence of Theorems 4.2 and 4.3 (the notation is from the previous section):

Corollary 5.1. (a) There exists a canonical isomorphism of $\Lambda$-algebras

$$
\Lambda\left[h_{1}, \ldots, h_{m-p}\right] /\left\langle E_{p+1}, \ldots, E_{m}\right\rangle \longrightarrow H_{T}^{*}(X),
$$

sending $h_{j}$ to $\sigma_{(j)}^{T}$ and where $s_{\lambda}$ is the equivariant Schubert class $\sigma_{\lambda}^{T}$.

(b) There exists a canonical isomorphism of $\Lambda$-algebras

$$
\Lambda\left[e_{1}, \ldots, e_{p}\right] /\left\langle H_{m-p+1}, \ldots, H_{m}\right\rangle \longrightarrow H_{T}^{*}(X),
$$

sending $e_{i}$ to $\sigma_{(1)^{i}}^{T}$ and $\widetilde{s}_{\lambda}$ to the equivariant Schubert class $\sigma_{\lambda}^{T}$.

Proof. It is known (see e.g. [23]) that there is a canonical isomorphism of $\Lambda$-algebras

$$
Q H_{T}^{*}(X) /\langle q\rangle \longrightarrow H_{T}^{*}(X)
$$

sending the equivariant quantum Schubert class $\sigma_{\lambda}$ from the previous section to $\sigma_{\lambda}^{T}$. Then the corollary follows from the Theorems 4.2 and 4.3.

Remarks. 1. The proof of the corollary can be given without using the equivariant quantum cohomology. There is an analogue of Proposition 3.2, stating that the Pieri-Chevalley rule determines the equivariant cohomology algebra. Then a 
"strictly equivariant" proof of Corollary 5.1 can be obtained by taking $q=0$ in all the assertions from the previous section.

2. The fact that the factorial Schur functions represent the equivariant Schubert classes can be also be deduced, indirectly, by combining the fact that the double Schubert polynomials represent the equivariant Schubert classes in the complete flag variety (cf. [6] and [1]) and that, when indexed by a Grassmannian permutation, these polynomials are actually factorial Schur functions. The latter holds because the vanishing property characterizing the factorial Schur functions ( $\S 2$, property (B)) is also satisfied by the double Schubert polynomials in question (see [20], p. 33). However, the details of this connection are missing from the literature.

3. It is well known that the equivariant Schubert classes are determined by their restriction to the torus fixed points in $X$. Formulae for such restrictions have been obtained by A. Knutson and T. Tao in [18] and, recently, by V. Lakshmibai, K.N. Raghavan, and P. Sankaran in [19].

5.1. A geometric interpretation of the factorial Schur functions. Consider the tautological short exact sequence on $X$ :

$$
0 \longrightarrow S \longrightarrow V \longrightarrow Q \longrightarrow 0
$$

which is clearly $T$-equivariant. Let $-x_{1}, \ldots,-x_{p}$ be the equivariant Chern roots of the bundle $S$. There is a weight space decomposition of the trivial (but not equivariantly trivial) vector bundle $V$ into a sum of $T$-equivariant line bundles:

$$
V=L_{1} \oplus \ldots \oplus L_{m} .
$$

Let $-T_{i}$ be the equivariant first Chern class of $L_{i} \cdot{ }^{3}$ Define the sequence $\left(t_{i}\right)$ as usual, using the formula from $\S 1.1$.

Proposition 5.2. In $H_{T}^{*}(X)$, the equivariant Schubert class $\sigma_{\lambda}^{T}$ is equal to the factorial Schur polynomial $s_{\lambda}(x \mid t)$.

Idea of proof. The equivariant Schubert class $\sigma_{\lambda}^{T}$ is a cohomology class on the infinite dimensional space $X_{T}$. The first step of the proof is to approximate this class by a class $\left(\sigma_{\lambda}\right)_{T, n}$ on a finite-dimensional "approximation" $X_{T, n}(n \gg 0)$ of $X_{T}$. This is standard (see e.g. [7] or [23]) and uses the $T$-bundle $\left(\mathbb{C}^{n+1} \backslash 0\right)^{m} \rightarrow\left(\mathbb{P}^{n}\right)^{m}$ which approximates the universal $T$-bundle $E T \rightarrow B T$. Then $X_{T, n}:=\left(E T_{n} \times X\right) / T$, which, in fact, is equal to the Grassmann bundle $\mathbb{G}\left(p, \mathcal{O}_{(1)}(-1) \oplus \ldots \oplus \mathcal{O}_{(m)}(-1)\right)$, where $\mathcal{O}_{(i)}(-1)$ denotes the tautological line bundle over the $i-$ th component of $\left(\mathbb{P}^{n}\right)^{m}$. Using this procedure one obtains the class $\left(\sigma_{\lambda}\right)_{T, n}$ as the cohomology class determined by the subvariety $\left(\Omega_{\lambda}\right)_{T, n}$ of $X_{T, n}$. The second step is to use the definition of $\left(\Omega_{\lambda}\right)_{T, n}$ to realize it as the degeneracy locus from [11], Thm. 14.3, whose cohomology class is given as a certain determinantal formula in the Chern classes of the vector bundles $S_{T, n}, V_{T, n}$ and $Q_{T, n}$ on $X_{T, n}$ induced by the tautological sequence on $X$. Finally, one proves that the determinant in question is equal to the claimed factorial Schur polynomial, which ends the proof.

\section{ACKNowledgements}

I am indebted to S. Fomin and W. Fulton whose comments enlightened the presentation of this paper. I am thankful to A. L. Mare and A. Yong for some

\footnotetext{
${ }^{3}$ All the minus signs are for positivity reasons. It turns out, for example, that $c_{1}^{T}\left(L_{i}\right)$ is the Chern class of $\mathcal{O}_{\mathbb{P}} \infty(-1)$ (see e.g. [24], §7).
} 
useful discussions and remarks. I would also like to thank the referee for providing valuable suggestions.

\section{REFERENCES}

[1] A. Arabia. Cohomologie T-èquivariante de la variété de drapeaux d'un groupe de Kac-Moody. Bull. Soc. Math. France, 117(2):129-165, 1989. MR1015806 (90i:32042)

[2] A. Astashkevich and V. Sadov. Quantum cohomology of partial flag manifolds $F_{n_{1}, \ldots, n_{k}}$. Commun. Math. Phys., 170:503-528, 1995. MR1337131 (96g:58027)

[3] A. Bertram. Quantum Schubert Calculus. Adv. Math., 128(2):289-305, 1997. MR1454400 (98j:14067)

[4] A. Bertram, I. Ciocan-Fontanine, and W. Fulton. Quantum multiplication of Schur polynomials. Journal of Algebra, 219(2):728-746, 1999. MR1706853 (2000k:14042)

[5] L. Biedenharn and J. Louck. A new class of symmetric polynomials defined in terms of tableaux. Advances in Applied Math., 10:396-438, 1989. MR1023942 (91c:05189)

[6] S. C. Billey. Kostant polynomials and the cohomology ring of G/B. Duke Math. J., 96:205224, 1999. MR1663931 (2000a:14060)

[7] M. Brion. Poincaré duality and equivariant (co)homology. Michigan Math. J. - special volume in honor of William Fulton, 48:77-92, 2000. MR1786481 (2001m:14032)

[8] A. S. Buch. Quantum cohomology of the Grassmannians. Compositio Math., 137(2):227-235, 2003. MR1985005 (2004c:14105)

[9] W. Chen and J. D. Louck. The factorial Schur function. J. of Math. Phys., 34(9):4144-4160, 1993. MR1233264 (95b:05210)

[10] D. Eisenbud. Commutative Algebra. With a view towards Algebraic Geometry. Graduate Texts in Mathematics, vol. 150. Springer-Verlag, New York, 1995. MR1322960 (97a:13001)

[11] W. Fulton. Intersection Theory. Springer-Verlag, 2nd edition, 1998. MR1644323 (99d:14003)

[12] A. Givental. Equivariant Gromov-Witten invariants. IMRN, (13):613-663, 1996. MR1408320 (97e:14015)

[13] A. Givental and B. Kim. Quantum cohomology of flag manifolds and Toda lattices. Comm. Math. Phys., 168:609-641, 1995. MR1328256 (96c:58027)

[14] I. Goulden and C. Greene. A new tableaux representation for supersymmetric Schur functions. Journal of Algebra, 170:687-703, 1994. MR1302864 (96f:05187)

[15] B. Kim. Quantum cohomology of partial flag manifolds and a residue formula for their intersection pairings. IMRN, (1):1-15, 1995. MR1317639 (96c:58028)

[16] B. Kim. On equivariant quantum cohomology. IMRN, (17):841-851, 1996. MR1420551 (98h:14013)

[17] B. Kim. Quantum cohomology of flag manifolds $G / B$ and quantum Toda lattices. Annals of Math., 149:129-148, 1999. MR1680543 (2001c:14081)

[18] A. Knutson and T. Tao. Puzzles and equivariant cohomology of Grassmannians. Duke Math. J., 119(2):221-260, 2003. MR1997946 (2006a:14088)

[19] V. Lakshmibai, K.N. Raghavan, and P. Sankaran. Equivariant Giambelli and determinantal restriction formulas for the Grassmannian. Pure Appl. Math. Q. 2(3):699-717, 2006. MR2252114 (2007h:14084)

[20] A. Lascoux. Interpolation - lectures at Tianjin University. June 1996.

[21] I. G. Macdonald. Schur functions, theme and variations. Actes 28éme Séminaire Lotharingien, pages 5-29, 1992. MR1308728 (95m:05245)

[22] I. G. Macdonald. Symmetric functions and Hall polynomials. With contributions by A. Zelevinsky. Oxford University Press, New York, 2nd edition, 1995. MR1354144 (96h:05207)

[23] L. C. Mihalcea. Equivariant quantum Schubert Calculus. Adv. of Math., 203(1):1-33, 2006. MR2231042 (2007c:14061)

[24] L. C. Mihalcea. Positivity in equivariant quantum Schubert calculus. Amer. J. of Math., 128(3):787-803, 2006. MR2230925

[25] A. I. Molev. Factorial supersymmetric Schur functions and super Capelli identities. In Proc. of the AMS - Kirillov's seminar on representation theory, pages 109-137, Providence, RI, 1998. Amer. Math. Soc.

[26] A. I. Molev and B. Sagan. A Littlewood-Richardson rule for factorial Schur functions. Trans. of Amer. Math. Soc., 351(11):4429-4443, 1999. MR1621694 (2000a:05212) 
[27] A. Okounkov. Quantum immanants and higher Capelli identities. Transformations Groups, 1:99-126, 1996. MR1390752 (97j:17010)

[28] A. Okounkov and O. Olshanski. Shifted Schur functions. St. Petersburg Math. J., 9(2), 1997. also available on ar $\chi$ iv: math: q-alg/9605042.

[29] A. Postnikov. Affine approach to quantum Schubert calculus. Duke Math. J., 128(3):473-509, 2003. MR2145741 (2006e:05182)

Department of Mathematics, Florida State University, 208 Love Building, TallahasSEE, FLORIDA 32312

E-mail address: mihalcea@math.fsu.edu

Current address: Department of Mathematics, Duke University, Box 90320, Durham, North Carolina 27708

E-mail address: lmihalce@math.duke.edu 